\title{
Loss of heterozygosity on tuberous sclerosis complex genes in multifocal micronodular pneumocyte hyperplasia
}

\author{
Takuo Hayashi ${ }^{1}$, Toshio Kumasaka ${ }^{2}$, Keiko Mitani ${ }^{1}$, Takashi Yao ${ }^{1}$, Koichi Suda ${ }^{1}$ and \\ Kuniaki Seyama ${ }^{3}$ \\ ${ }^{1}$ Department of Human Pathology, Juntendo University School of Medicine, Tokyo, Japan; ${ }^{2}$ Department of \\ Pathology, Japanese Red Cross Medical Center, Tokyo, Japan and ${ }^{3}$ Department of Respiratory Medicine, \\ Juntendo University School of Medicine, Tokyo, Japan
}

\begin{abstract}
Multifocal micronodular pneumocyte hyperplasia is a rare pulmonary manifestation of tuberous sclerosis complex (TSC) that is a tumor suppressor gene disorder characterized by many hamartomas. A purported mechanism of hamartomatous proliferation in TSC is constitutive activation of the mammalian target of rapamycin (mTOR) signaling pathway dysregulated by a functional loss of TSC genes. Although multifocal micronodular pneumocyte hyperplasia develops locally as self-limited, benign lesions, it is morphologically similar to the preinvasive lesion of pneumocytes that characterize atypical adenomatous hyperplasia or bronchioloalveolar carcinoma. Frequently both conditions include a loss of heterozygosity on TSC. The goal of this study was to determine whether multifocal micronodular pneumocyte hyperplasia is neoplastic. Loss of heterozygosity on TSC genes and immunohistochemistry for mTOR-related proteins (phospho-mTOR, phospho-p70S6K, phospho-S6, and phospho-Akt) were analyzed in 42 lesions: 16 multifocal micronodular pneumocyte hyperplasia (7 patients with TSC, 1 TSC not confirmed), 14 atypical adenomatous hyperplasia, and 12 bronchioloalveolar carcinoma (9 and 12 patients, respectively). The results showed that at least one of two multifocal micronodular pneumocyte hyperplasia lesions from each patient had loss of heterozygosity on TSC1 or TSC2 (15 or 50\%) and were frequently immunopositive for phospho-mTOR (88\%), phospho-p70S6K (100\%), and phospho-S6 (100\%) but not phospho-Akt (14\%), an upstream regulatory protein of mTOR. Loss of heterozygosity of TSC was found in the preinvasive lesions of pneumocytes, equal to or less than multifocal micronodular pneumocyte hyperplasia. In contrast, phospho-Akt was expressed in the preinvasive lesions of pneumocytes more frequently than multifocal micronodular pneumocyte hyperplasia, but the other mTOR-related proteins were less frequently expressed in the former than in the latter. These outcomes suggest that functional loss of TSCs and consequent hyperphosphorylation of mTOR-related proteins in multifocal micronodular pneumocyte hyperplasia may cause its benign neoplastic proliferation of pneumocytes.
\end{abstract}

Modern Pathology (2010) 23, 1251-1260; doi:10.1038/modpathol.2010.114; published online 4 June 2010

Keywords: loss of heterozygosity; lung adenocarcinoma; mTOR; multifocal micronodular pneumocyte hyperplasia; tuberous sclerosis complex

Patients with phakomatoses frequently develop benign lesions that rarely progress to malignant transformation, that is, hamartomas. ${ }^{1}$ Individuals with tuberous sclerosis complex (TSC), one of the

Correspondence: Dr T Kumasaka, MD, PhD, Department of Pathology, Japanese Red Cross Medical Center, 4-1-22, Hiroo, Shibuya-ku, Tokyo 150-8935, Japan.

E-mail: kumasaka_toshio@med.jrc.or.jp

Received 4 March 2010; revised and accepted 13 April 2010; published online 4 June 2010 phakomatoses, also have hamartomas throughout the body presenting as cortical tubers and subependymal nodules, angiofibromas, cardiac rhabdomyomas, subungual fibromas, renal angiomyolipomas, or pulmonary lymphangioleiomyomatosis. TSC is caused by a mutation of TSC1 or TSC2 genes located at chromosome $9 \mathrm{q}$ or $16 \mathrm{p}$, respectively, known as tumor suppressor genes. ${ }^{2,3}$ For renal angiomyolipomas and pulmonary lymphangioleiomyomatosis, the pathogenic mechanism of hamartomatous development has been attributed to a functional loss of 
TSC genes, causing the benign neoplastic proliferation of somatic cells through constitutive activation of the mammalian target of rapamycin (mTOR) signaling pathway, which has a crucial role in cell growth, proliferation, and metabolism. ${ }^{4-6}$ Popper et al in $1991^{7}$ then others ${ }^{8-21}$ reported multifocal micronodular pneumocyte hyperplasia in the lungs of patients with TSC. Multifocal micronodular pneumocyte hyperplasia is a rare lung disease usually found in young or middle-aged women with TSC but uncommonly diagnosed because few of these patients have multifocal micronodular pneumocyte hyperplasia-related symptoms. ${ }^{22}$ However, high-resolution computed tomography shows small nodules with ground grass opacity throughout the lung parenchyma. The histology is characterized by numerous, hyperplastic lesions of large type II pneumocytes similar to atypical adenomatous hyperplasia of the lung and bronchioloalveolar carcinoma. To date, only two clinicopathological analyses or meta-analyses for multifocal micronodular pneumocyte hyperplasia were performed and both yielded similar conclusions: (1) a causal relationship between multifocal micronodular pneumocyte hyperplasia and TSC, (2) the hamartomatous nature of multifocal micronodular pneumocyte hyperplasia, and (3) a morphological similarity between atypical adenomatous hyperplasia and bronchioloalveolar carcinoma. ${ }^{11,21}$ Recently, Takamochi et $a l^{23}$ reported that loss of heterozygosity $(\mathrm{LOH})$ on $9 \mathrm{q}$ and $16 \mathrm{p}$ as TSC gene-associated loci often appeared in multiple lesions of atypical adenomatous hyperplasia and lung adenocarcinomas. Conceivably, then, TSC gene-associated loci might be a mechanism of pneumocyte transformation into atypical adenomatous hyperplasia and adenocarcinomas in the lungs of patients even without TSC. Speculation followed that multifocal micronodular pneumocyte hyperplasia may be neoplastic and share a common pathogenic mechanism of not only TSC-related tumors but also alveolar epithelial neoplastic lesions. However, whether multifocal micronodular pneumocyte hyperplasia has LOH on TSC genes and depletes their function is unclear.

Here, we studied the LOH of TSC genes and immunohistochemistry for the mTOR signaling pathway in seven TSC females with multifocal micronodular pneumocyte hyperplasia and one with an unknown TSC status. We identified LOH on TSC genes in all these patients more frequently than in those with atypical adenomatous hyperplasia or bronchioloalveolar carcinoma. Immunohistochemistry revealed hyperphosphorylation of mTOR-related proteins but not an upstream regulatory protein, p-Akt, of the mTOR signaling pathway in multifocal micronodular pneumocyte hyperplasia cells. These results indicated that development of multifocal micronodular pneumocyte hyperplasia cells, but not necessarily preinvasive lesions of pneumocytes, might require constitutive activation of the mTOR signaling pathway stemming from the loss of TSC gene function.

\section{Materials and methods}

\section{Patients}

The clinical features of patients with multifocal micronodular pneumocyte hyperplasia, atypical adenomatous hyperplasia of the lung, and bronchioloalveolar carcinoma reviewed here are summarized in Table 1. Six surgical samples (M1-5 and M8) and two from autopsies (M6 and M7) of eight patients with multifocal micronodular pneumocyte hyperplasia were provided by Iwate Prefectural Central Hospital, Nishinouchi Branch of Ohta General Hospital, International Medical Center of Japan, Okayama University Hospital, and Himeji

Table 1 Clinicopathological feature of patients with multifocal micronodular pneumocyte hyperplasia, atypical adenomatous hyperplasia, and bronchioloalveolar carcinoma

\begin{tabular}{|c|c|c|c|c|c|c|}
\hline Patient & $A g e^{\mathrm{a}}$ & Gender & TSC & $L A M$ & $\begin{array}{l}\text { Adenocar- } \\
\text { cinoma }^{\mathrm{b}}\end{array}$ & $\begin{array}{c}\text { Tumor } \\
\text { size }(\mathrm{mm})\end{array}$ \\
\hline
\end{tabular}

\begin{tabular}{ccccccc}
\hline \multicolumn{7}{c}{ Multifocal micronodular pneumocyte hyperplasia } \\
M1 & 30 & F & + & + & - & 5 \\
M2 & 41 & F & + & - & - & 5 \\
M3 & 45 & F & + & + & - & 4 \\
M4 & 53 & F & + & + & - & 3 \\
M5 & 54 & F & NA $^{\mathrm{c}}$ & - & - & 2 \\
M6 & 57 & F & + & - & - & 5 \\
M7 & 62 & F & + & - & - & 3 \\
M8 $^{\text {d }}$ & 64 & F & + & + & + & 2
\end{tabular}

Atypical adenomatous hyperplasia

$\begin{array}{lllllll}\text { A1 } & 40 & \text { F } & - & - & - & 1 \\ \text { A2 } & 54 & \text { M } & - & - & + & 3 \\ \text { A3 } & 61 & \text { F } & - & - & + & 2 \\ \text { A4 } & 62 & \text { F } & - & - & + & 6 \\ \text { A5 } & 67 & \text { F } & - & - & + & 2 \\ \text { A6 } & 70 & \text { F } & - & - & + & 2 \\ \text { A7 } & 70 & \text { F } & - & - & - & 4 \\ \text { A8 } & 79 & \text { F } & - & - & + & 3 \\ \text { A9 } & 80 & \text { F } & - & - & + & 7\end{array}$

Bronchioloalveolar carcinoma

$\begin{array}{llllllr}\text { B1 } & 45 & \text { F } & - & - & - & 9 \\ \text { B2 } & 59 & \text { F } & - & - & - & 9 \\ \text { B3 } & 62 & \text { M } & - & - & - & 29 \\ \text { B4 } & 58 & \text { F } & - & - & - & 12 \\ \text { B5 } & 64 & \text { M } & - & - & - & 17 \\ \text { B6 } & 70 & \text { M } & - & - & - & 13 \\ \text { B7 } & 72 & \text { F } & - & - & & 22 \\ \text { B8 } & 73 & \text { F } & - & - & + & 10 \\ \text { B9 } & 78 & \text { F } & - & - & + & 7 \\ \text { B10 } & 78 & \text { M } & - & - & + & 15 \\ \text { B11 } & 79 & \text { F } & - & - & + & 5 \\ \text { M8 }^{d} & 64 & \text { F } & + & + & + & 5\end{array}$

LAM, lymphangioleiomyomatosis; TSC, tuberous sclerosis complex. ${ }^{\mathrm{a}}$ The age of the patient at which the examination was performed.

${ }^{\mathrm{b}}$ The concomitant invasive pulmonary adenocarcinoma.

${ }^{\mathrm{C}}$ Not available because of limited clinical dates.

${ }^{\mathrm{d}}$ Lesions of multifocal micronodular pneumocyte hyperplasia and bronchioloalveolar carcinoma were detected in the same patient. 
Medical Center. All patients with multifocal micronodular pneumocyte hyperplasia were female; one (M3) had a TSC2 germ-line mutation, ${ }^{14}$ and the TSC status of the others was clinically confirmed with one exception (M5). Of the seven multifocal micronodular pneumocyte hyperplasia patients with TSC, four had pulmonary lymphangioleiomyomatosis. One (M8) had pulmonary adenocarcinomas including bronchioloalveolar carcinoma. In two, multifocal micronodular pneumocyte hyperplasia was incidentally found at autopsy. For comparison to multifocal micronodular pneumocyte hyperplasia, 9 surgical materials of atypical adenomatous hyperplasia patients and 11 of nonmucinous bronchioloalveolar carcinoma were obtained from the archives of the Department of Human Pathology at Juntendo University from 2001 to 2007. No patients from Juntendo University with atypical adenomatous hyperplasia or bronchioloalveolar carcinoma had either TSC or pulmonary lymphangioleiomyomatosis. Eight of 9 (89\%) patients with atypical adenomatous hyperplasia and 8 of $12(67 \%)$ with bronchioloalveolar carcinoma were female. Of the atypical adenomatous hyperplasia group, one (A7) had multiple atypical adenomatous hyperplasia lesions. Seven of nine $(78 \%)$ patients with atypical adenomatous hyperplasia simultaneously had adenocarcinomas of the lung. The pathological characteristics of multifocal micronodular pneumocyte hyperplasia, atypical adenomatous hyperplasia, and bronchioloalveolar carcinoma (Figure 1) were assessed by two pathologists (TH and TK) following the previous reports. ${ }^{11,23,24}$

\section{Microdissection and DNA Extraction}

All tissues were fixed in $10 \%$ buffered formalin, embedded in paraffin after routine processing, sectioned at $4 \mu \mathrm{m}$, and stained with hematoxylin and eosin and elastica-Masson's trichrome. Specimens (8- $\mu \mathrm{m}$ thick) were sectioned from each block for microdissection. The specimens were stained with cytokeratin (CK) (Kl-1; Immunotech, Marseille, France), following the immunohistochemical method described below and counterstained with hematoxylin. To eliminate contamination during microdissection, we irradiated the CK-negative cells, including interstitial cells, lymphocytes, and macrophages, in at least $2 \mathrm{~mm}^{2}$ area of lesions using a Laser Microdissection System (Leica Microsystems, Wetzlar, Germany). Then, CK-positive cells in the laser-radiated area, that is, multifocal micronodular pneumocyte hyperplasia cells, atypical adenomatous hyperplasia cells, or bronchioloalveolar carcinoma cells, were scraped out with a 27-gauge needle. A normal lung tissue sample from each patient was also scraped as a normal control. DNA from the scraped tissues was extracted with $25 \mu \mathrm{l}$ extraction buffer consisting of $50 \mathrm{mM}$ Tris-HCl (pH 8.0), $1 \mathrm{mM}$ EDTA, and $0.5 \%$ Tween 20 , and 1:50 volume of proteinase $\mathrm{K}$ solution $(10 \mathrm{mg} / \mathrm{ml})$.

\section{Loss of Heterozygosity Analyses}

We analyzed LOH on chromosome 9q (including the TSC1 gene-associated region) and 16q (including the TSC2 gene-associated region) using the following 12 microsatellite markers: D9S149, D9S2126, D9S1830, D9S1199, S9S1198, D9S150, D16S521, D16S525, Kg8, D16S291, D16S663, and D16S283. PCR was performed in a $15 \mu \mathrm{l}$ volume of a mixture containing $1.5 \mu \mathrm{l}$ GeneAmp $10 \times$ PCR Buffer II, $1.5 \mu \mathrm{l} 2 \mathrm{mM}$ dNTP, $1.5-2.5 \mathrm{mM} \mathrm{MgCl}_{2}, 0.12 \mu \mathrm{l}$ of $5 \mathrm{U} / \mu \mathrm{l}$ Ampli Taq Gold (all from Applied Biosystems, Foster City, CA, USA), 7.38-7.98 $\mu \mathrm{l} \mathrm{H}_{2} \mathrm{O}, 1.0 \mu \mathrm{l}$ each primer, and $1.0 \mathrm{ml}$ sample. The mixture was heated at $95^{\circ} \mathrm{C}$ for $6 \mathrm{~min}$ and then cycled 40 times in a Program Temp Control System (ASTEC, Tokyo, Japan). Each cycle consisted of $30 \mathrm{~s}$ at $94^{\circ} \mathrm{C}$ for denaturing, $30 \mathrm{~s}$ at $57-60^{\circ} \mathrm{C}$ for annealing, $60 \mathrm{~s}$ at $72^{\circ} \mathrm{C}$ for elongation, and $10 \mathrm{~min}$ at $72^{\circ} \mathrm{C}$ for final elongation. The samples were electrophoresed using an Applied Biosystems 3130/3130xl Genetic Analyzer or Applied Biosystems 377 DNA Sequencer. LOH analysis was performed using a Gene Mapper 4.0 or a Gene Scan 3.1 (both from Applied Biosystems). To confirm reproducibility, all lesions were examined at least twice. A reduction in signal intensity over $50 \%$ was defined as LOH. Because we did not obtain adequate DNA from several multifocal micronodular pneumocyte hyperplasia samples, we did not analyze $\mathrm{LOH}$ on chromosome $9 \mathrm{q}$ in two such patients (lesions 4 and 8) or at some microsatellite markers including D9S2126, D9S1830, D9S150, D16S521, and D16S663 in five lesions (lesions 3, 7, 9, 11, and 12), three microsatellite markers including D9S2126, D9S1830, and D9S150 in lesion 10 , and at two microsatellite markers including D16S521 and D16S663 in lesion 8.

\section{Immunohistochemical Examination}

Four-sectioned paraffin-embedded tissues were deparaffinized and hydrated. For antigen retrieval, sections were heated in $1 \mathrm{mM}$ EDTA ( $\mathrm{pH}$ 8.0) or citrate buffer ( $\mathrm{pH}$ 6.0) for 5-30 min. Endogenous peroxidase activity was blocked with $0.3 \%$ hydrogen peroxide in methanol at room temperature. Immunohistochemical examinations were carried out with the EnVision + System (DakoCytomation, Glostrup, Denmark) using rabbit polyclonal antibodies against phospho (p)-Akt (Ser473; Cell Signaling Technology, Beverly, MA, USA), p-mTOR (Ser2448; Cell Signaling Technology), p-p70S6K (Thr389; Cell Signaling Technology), and p-S6 (Ser235/236; Cell Signaling Technology). Immunopositivity was scored as 0 , undetectable or heterogeneous positivity for $<5 \%$ of tumor cells; $1+$, strong positivity in $5-30 \%$ of tumor cells; $2+$, strong positivity in $30-70 \%$ of tumor cells; and $3+$, diffuse positivity in more than $70 \%$ of tumor cells. 

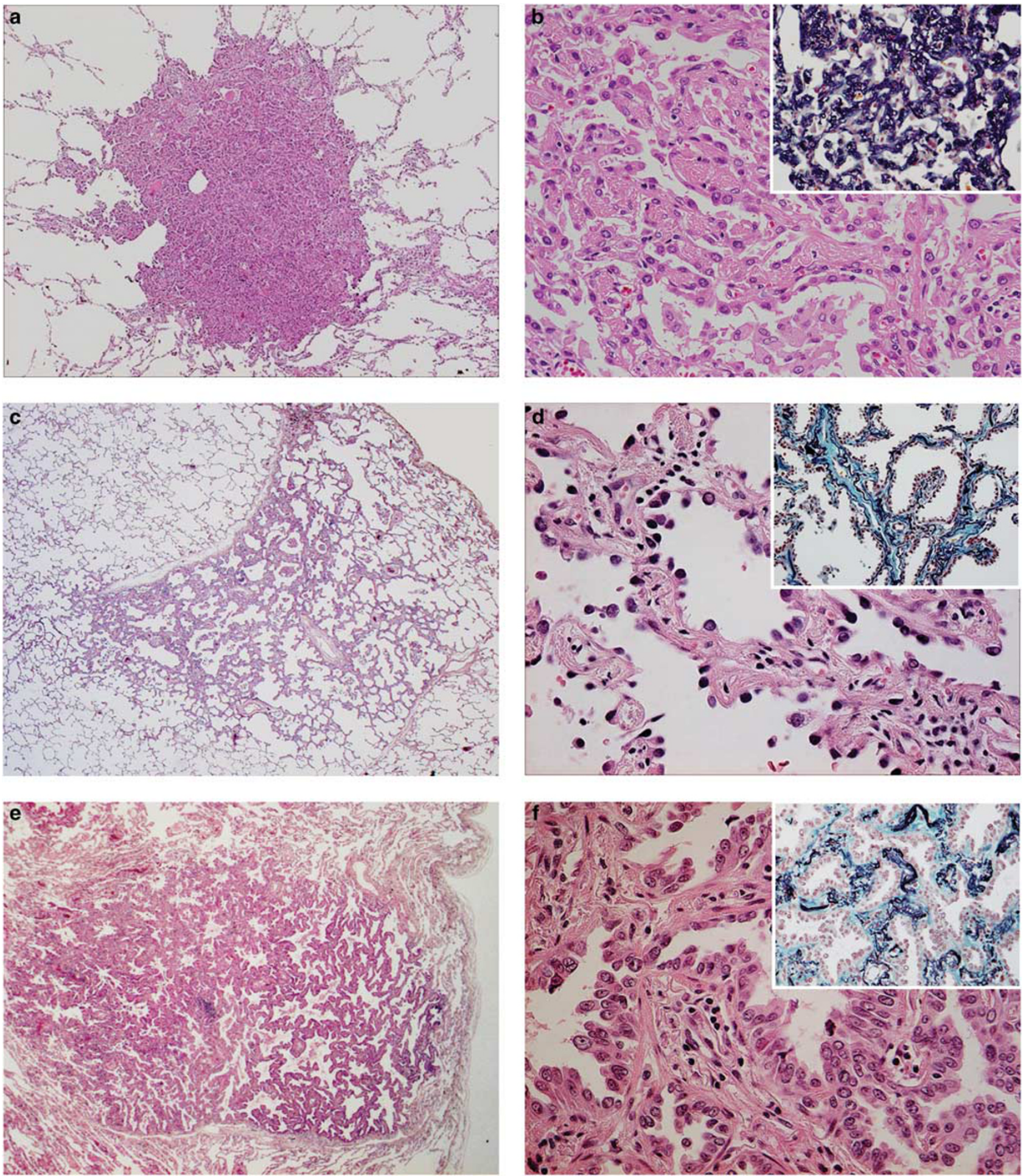

Figure 1 Representative histopathological findings of multifocal micronodular hyperplasia, atypical adenomatous hyperplasia, and bronchioloalveolar carcinoma. In the low-power view, multifocal micronodular hyperplasia is well-demarcated, nodular lesions ranging 2-5 $\mathrm{mm}$ in pulmonary parenchyma (a). In the high-power view, enlarged cuboidal cells lining the thickened alveolar septa had abundant, eosinophilic cytoplasm and large, round nuclei with nucleoli lacking mitosis. Airspaces of the lesions were filled with those cells and alveolar macrophages. The thickened alveolar septa were composed of markedly thickened elastic fibers and infiltrated by many lymphocytes (b (inset), elastica-Masson's trichrome stain). In the low-power view, atypical adenomatous hyperplasia (c) and bronchioloalveolar carcinoma (e) were less demarcated than multifocal micronodular hyperplasia. In the high-power view, atypical cuboidal cells of both proliferated along the alveolar septa and had large, round or oval nuclei with higher nuclear/cytoplasmic ratio than multifocal micronodular hyperplasia. The elastic fibers of both lesions were less prominent than in multifocal micronodular hyperplasia (d and f (inset), elastica-Masson's trichrome stain). Cells in bronchioloalveolar carcinoma lesions had more well-defined boundaries with thickened alveolar septa and had larger nuclei than those of atypical adenomatous hyperplasia. 


\section{Statistical Analyses}

Kruskal-Wallis $H$-test and Dunn's test, or Fisher's exact test (StatMate III for Windows; ATMS, Tokyo, Japan) were used for statistical analysis. A $P$-value $<0.05$ was considered significant.

\section{Results}

\section{Loss of Heterozygosity Analyses}

We examined 16 multifocal micronodular pneumocyte hyperplasia lesions for chromosome 16p from eight patients and 13 multifocal micronodular pneumocyte hyperplasia lesions for chromosome $9 p$ from seven patients. A representative example of $\mathrm{LOH}$ is shown in Figure 2. The distribution of deletions is shown in Figure 3. At least one of two multifocal micronodular pneumocyte hyperplasia lesions had LOH on $16 \mathrm{p}$ in seven of eight patient $(88 \%)$ and $\mathrm{LOH}$ on $9 \mathrm{q}$ in one patient $(12 \%)$. In multifocal micronodular pneumocyte hyperplasia lesions, $\mathrm{LOH}$ on $16 \mathrm{p}$ or $9 \mathrm{q}$ was detected in 8 of 16 lesions $(50 \%)$ or 2 of 13 lesions (15\%), respectively. No lesion had LOH on both 9q and 16p. Two of eight patients had LOH of both lesions: one pair had the
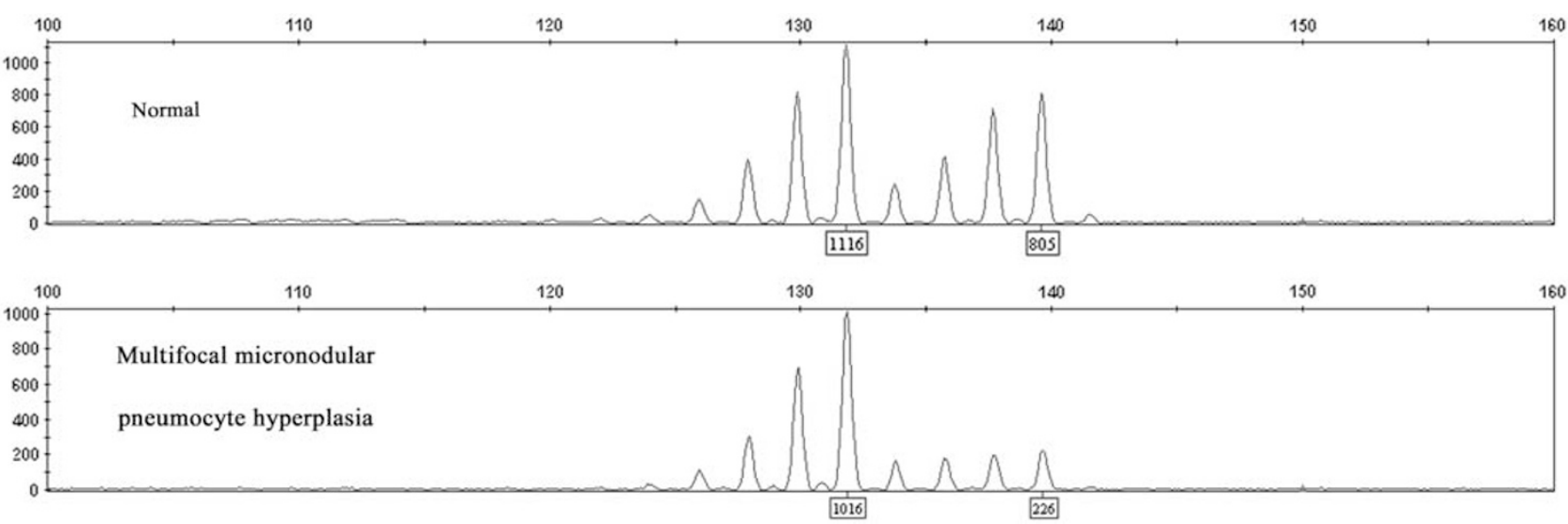

Figure 2 Representative example of microsatellite analysis. Patient M8 (lesion 15) shows loss of heterozygosity (LOH) at D9S2126.

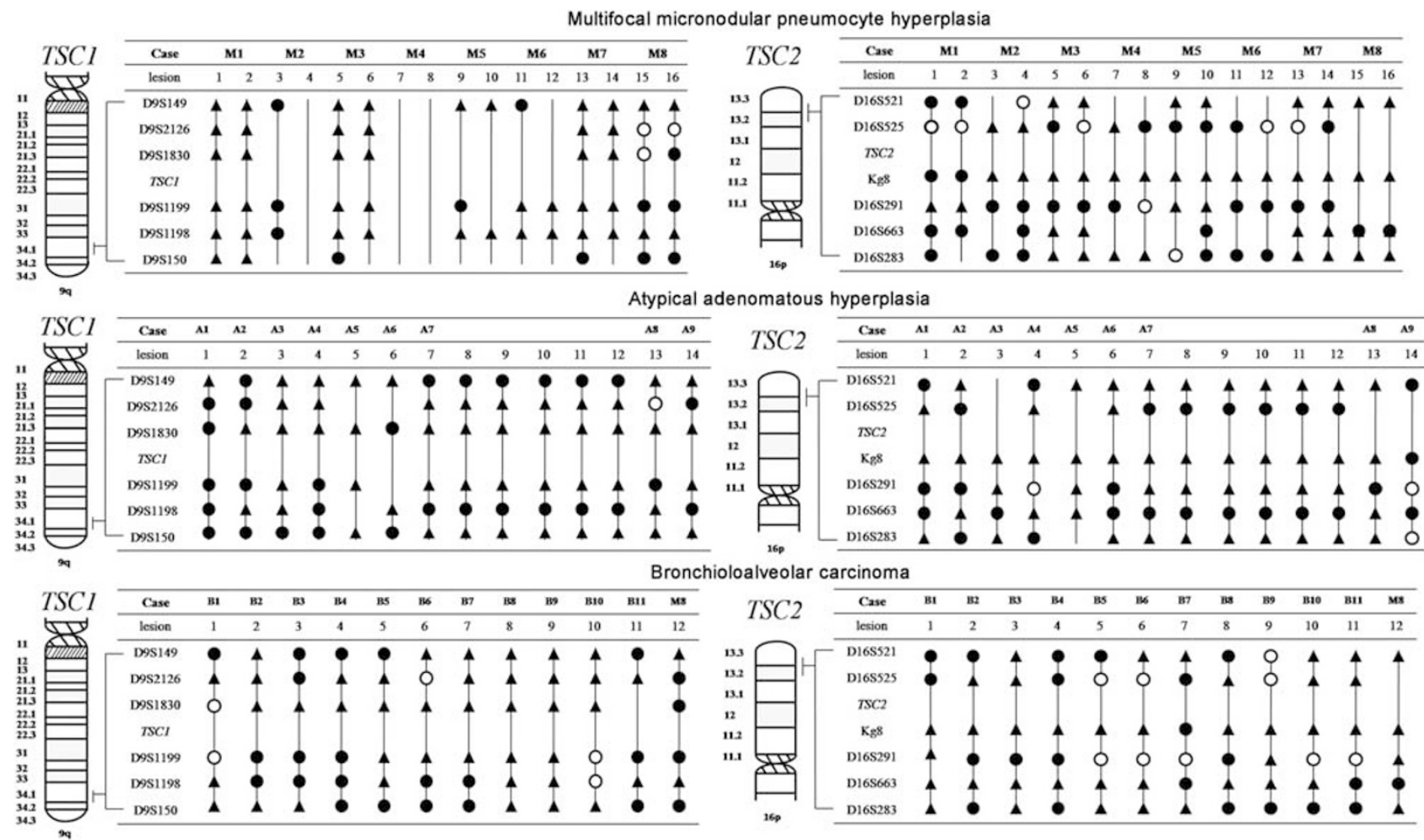

Figure 3 The distribution of deletions on 16p including the TSC2 gene-associated region and 9p including the TSC1 gene-associated region in multifocal micronodular hyperplasia, atypical adenomatous hyperplasia, and bronchioloalveolar carcinoma. Open circle indicates loss of heterozygosity $(\mathrm{LOH})$, closed circle indicates retention of heterozygosity, and black triangle indicates uninformative. No symbol indicates that LOH analysis was not examined at the loci. 
same pattern of LOH and the other did not. Of 10 lesions with $\mathrm{LOH}, 1$ had two loci of $\mathrm{LOH}$, which were held in 9q. To compare to multifocal micronodular pneumocyte hyperplasia, we examined 14 atypical adenomatous hyperplasia lesions from 9 patients and 12 bronchioloalveolar carcinoma lesions from 12 patients, including 1 patient with multifocal micronodular pneumocyte hyperplasia (M8) for $\mathrm{LOH}$ on 9q and 16p. In atypical adenomatous hyperplasia, LOH on TSCs was detected in three of nine patient samples (33\%). Two of 14 lesions showed LOH on 16p (14\%), and 1 of 14 lesions showed $\mathrm{LOH}$ on $9 \mathrm{q}(7 \%)$. One lesion had $\mathrm{LOH}$ at two loci in 16p, and the other two had $\mathrm{LOH}$ at one locus in $9 \mathrm{q}$ or $16 \mathrm{p}$. However, no lesion had $\mathrm{LOH}$ on both $16 \mathrm{p}$ and 9q. In bronchioloalveolar carcinoma, $\mathrm{LOH}$ was detected in 7 of 12 patient samples $(58 \%)$, which was more than that in the atypical adenomatous hyperplasia group. Six of 12 lesions $(50 \%)$ from a bronchioloalveolar carcinoma source had LOH on $16 \mathrm{p}$, and 3 of 12 lesions $(25 \%)$ had $\mathrm{LOH}$ on 9q. A lesion obtained from bronchioloalveolar carcinoma patient M8 had no LOH.

\section{Immunohistochemistry}

As illustrated in Figure 4 and summarized in Tables 2 and 3, multifocal micronodular pneumocyte hyperplasia cells were immunopositive for p-p70S6K in seven of seven patients $(100 \%)$, for p-S6 in eight of eight patients $(100 \%)$, and for p-mTOR in seven of eight patients (88\%). Meanwhile, only one of seven patients $(14 \%)$ had a few multifocal micronodular pneumocyte hyperplasia cells that were immunopositive for p-Akt. In contrast to multifocal micronodular pneumocyte hyperplasia, atypical adenomatous hyperplasia, and bronchioloalveolar carcinoma lesions exhibited a high frequency of immunopositivity for p-Akt in 7 of 9 patients $(78 \%)$ and 10 of 12 patients (83\%), respectively. Atypical adenomatous hyperplasia was associated with immunopositivity for p-mTOR in eight of nine patients $(89 \%)$ with p-p70S6K, and with p-S6 in five of nine patients (56\%). However, for bronchioloalveolar carcinoma, p-mTOR immunopositivity was less frequent in 6 of $12(50 \%)$ and p-p70S6K or p-S6 in 3 of 12 (25\%) patients than for each of those proteins in patients with atypical adenomatous hyperplasia or multifocal micronodular pneumocyte hyperplasia. The frequencies of immunopositivity for all but p-mTOR were statistically significant between multifocal micronodular pneumocyte hyperplasia and bronchioloalveolar carcinoma (p-AKT, $P<0.01$; p-S6, $P<0.05$; and p-p70S6K, $P<0.05$ ). In the correlation between LOH and immunohistochemistry, seven of eight patients in the multifocal micronodular pneumocyte hyperplasia group having $\mathrm{LOH}$ showed immunopositivity for all proteins of the mTOR signaling pathway; meanwhile, one of seven lesions of bronchioloalveolar carcinoma and one of three of atypical adenomatous hyperplasia showed immunopositivity for all the proteins.

\section{Discussion}

With this study initiated to address the possibility that multifocal micronodular pneumocyte hyperplasia is a neoplastic disease, we showed for the first time that all of eight patients with multifocal micronodular pneumocyte hyperplasia lost heterozygosity of TSC genes: in seven TSC2 was affected and in one TSC1. One of us (KS) previously described a patient (M3) with a TSC2 germ-line mutation and associated $\mathrm{LOH}$ in a lesion of pulmonary lymphangioleiomyomatosis, but not in a lesion of multifocal micronodular pneumocyte hyperplasia. ${ }^{14}$ Possibly, that apparent absence of $\mathrm{LOH}$ on the TSC2 gene resulted from contamination of normal cells by infiltrating inflammatory cells in the multifocal micronodular pneumocyte hyperplasia lesions. ${ }^{11}$ Alternatively, the relatively low (50\%) detection rate for $\mathrm{LOH}$ on TSC2 in multifocal micronodular pneumocyte hyperplasia lesions even in this series might has been the cause. Thus, it remained unclear whether the TSC2 gene was involved in multifocal micronodular pneumocyte hyperplasia. However, results from this study clearly showed that one of two multifocal micronodular pneumocyte hyperplasia lesions obtained from the same patient had LOH on TSC2. Because $\mathrm{LOH}$ on TSC genes is consistent with loss of the wild-type allele, Knudson's two-hit tumor suppressor gene theory may apply to the multifocal micronodular pneumocyte hyperplasia lesion of this patient as well as to those of lymphangioleiomyomatosis or angiomyolipoma in patients with TSC. $^{25,26}$ Thus, it indicated that at least a multifocal micronodular pneumocyte hyperplasia lesion associated with LOH on TSC2 had lost both alleles of TSC2, strongly indicating that multifocal micronodular pneumocyte hyperplasia might be neoplastic.

Because multifocal micronodular pneumocyte hyperplasia may be neoplastic, the question arises whether multifocal micronodular pneumocyte hyperplasia consists of genetically independent tumors. Patients with lymphangioleiomyomatosis, a major pulmonary manifestation among TSC-associated hamartomas, have multiple lesions in the lungs as well as multifocal micronodular pneumocyte hyperplasia. Because those afflicted with lymphangioleiomyomatosis have identical $\mathrm{LOH}$ patterns in the lungs and lymph nodes, lymphangioleiomyomatosis has been considered as a benign metastatic tumor. ${ }^{25-28}$ We detected $\mathrm{LOH}$ in both multifocal micronodular pneumocyte hyperplasia lesions in only two patients: in one patient the $\mathrm{LOH}$ pattern was identical between the lesions and in the other it was not. Meanwhile, the other six patients had two lesions with and without LOH. There were 

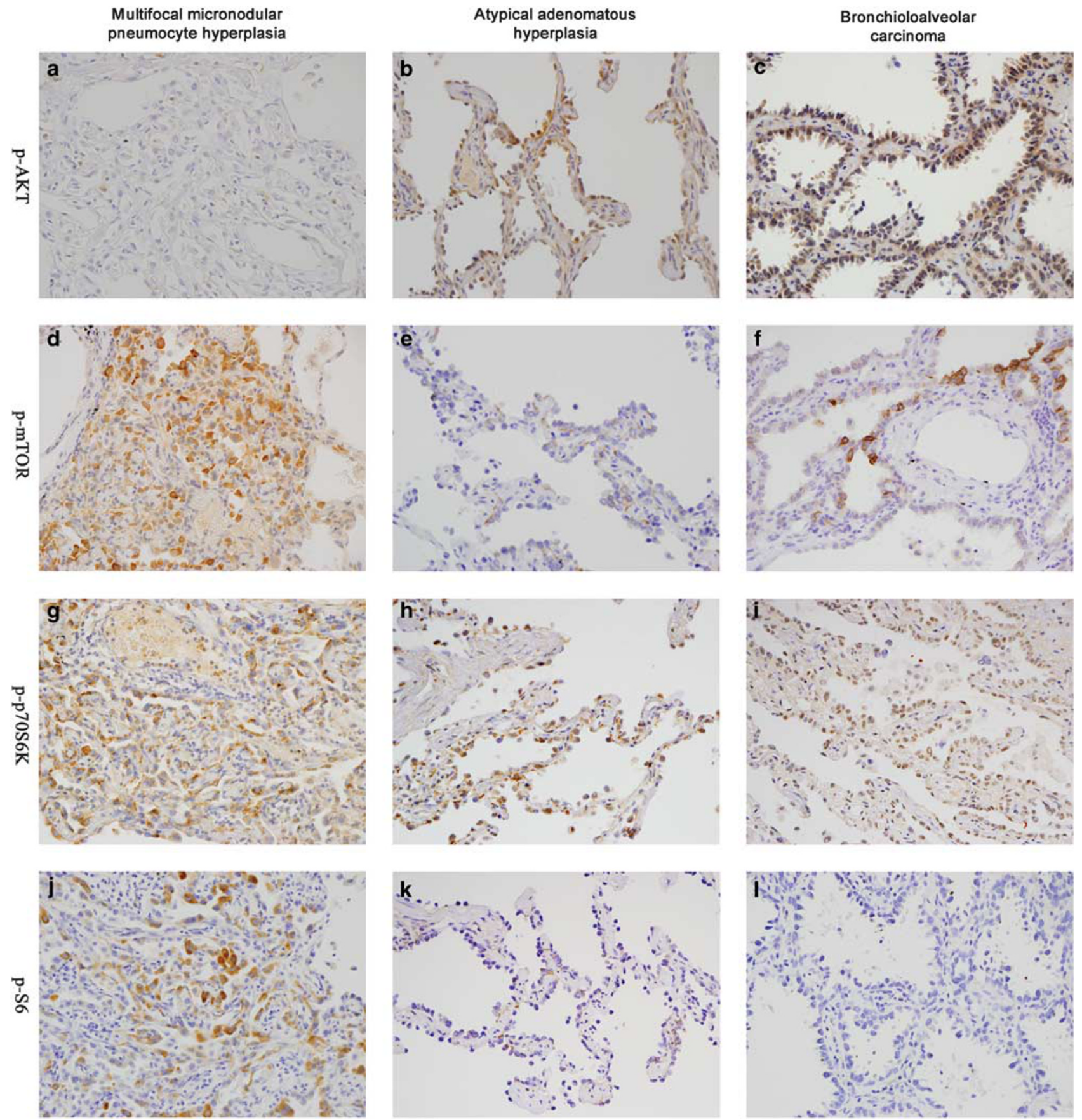

Figure 4 Representative results of immunohistochemical examination of lesions from patients with multifocal micronodular hyperplasia, atypical adenomatous hyperplasia, and bronchioloalveolar carcinoma. Negative staining in multifocal micronodular hyperplasia, $2+$ nuclear and cytoplasmic staining in atypical adenomatous hyperplasia, and $3+$ nuclear and cytoplasmic staining in bronchioloalveolar carcinoma of p-AKT (a, b, and $\mathbf{c}$, respectively). $3+$ cytoplasmic staining in multifocal micronodular hyperplasia, $1+$ cytoplasmic staining in atypical adenomatous hyperplasia, and $1+$ cytoplasmic staining in bronchioloalveolar carcinoma of p-mTOR (d, e, and f, respectively). $3+$ cytoplasmic staining in multifocal micronodular hyperplasia, $3+$ cytoplasmic staining in atypical adenomatous hyperplasia, and $2+$ nuclear staining in bronchioloalveolar carcinoma of p-p70S6K (g, h, and $\mathbf{i}$, respectively). $2+$ cytoplasmic staining in multifocal micronodular hyperplasia, $1+$ cytoplasmic staining in atypical adenomatous hyperplasia, and negative staining in bronchioloalveolar carcinoma of p-S6 (j, $\mathbf{k}$, and $\mathbf{l}$, respectively).

four possibilities for no LOH of multifocal micronodular pneumocyte hyperplasia: (1) some lesions of multifocal micronodular pneumocyte hyperplasia might consist of reactive pneumocytes, (2) the markers for $\mathrm{LOH}$ might be not informative, (3) multifocal micronodular pneumocyte hyperplasia might have an epigenetic alteration in TSC genes, and (4) samples of multifocal micronodular pneumocyte hyperplasia might be contaminated with normal cells. First, it was unlikely that multifocal micronodular pneumocyte hyperplasia patients would bear combined subpopulations of neoplastic 
Table 2 Results of LOH analysis and immunohistochemistry for AKT-mTOR signaling pathway

\begin{tabular}{|c|c|c|c|c|c|}
\hline Patient & $\mathrm{LOH}$ & $p-A K T$ & $p-m T O R$ & $p-p 70 S 6 K$ & $p-S 6$ \\
\hline \multicolumn{6}{|c|}{ Multifocal micronodular pneumocyte hyperplasia } \\
\hline M1 & TSC2 & - & +++ & +++ & + \\
\hline M2 & TSC2 & - & +++ & + & + \\
\hline M3 & TSC2 & - & + & +++ & ++ \\
\hline M4 & TSC2 & - & +++ & + & + \\
\hline M5 & TSC2 & - & +++ & + & +++ \\
\hline M6 & TSC2 & $\mathrm{NE}$ & - & NE & + \\
\hline M7 & TSC2 & + & +++ & ++ & +++ \\
\hline M8 & TSC1 & - & +++ & ++ & + \\
\hline \multicolumn{6}{|c|}{ Atypical adenomatous hyperplasia } \\
\hline A1 & - & +++ & +++ & +++ & ++ \\
\hline A2 & - & - & +++ & - & - \\
\hline A3 & - & ++ & + & +++ & - \\
\hline A4 & TSC2 & - & + & - & - \\
\hline A5 & - & + & - & - & - \\
\hline A6 & - & ++ & ++ & +++ & ++ \\
\hline A7 & - & ++ & +++ & +++ & ++ \\
\hline A8 & TSC1 & + & ++ & ++ & + \\
\hline A9 & TSC2 & ++ & + & - & + \\
\hline \multicolumn{6}{|c|}{ Bronchioloalveolar carcinoma } \\
\hline B1 & TSC1 & - & - & - & - \\
\hline B2 & - & +++ & - & - & - \\
\hline B3 & - & $++t$ & - & - & - \\
\hline B4 & - & +++ & + & - & - \\
\hline B5 & TSC2 & +++ & + & - & - \\
\hline B6 & TSC1 and 2 & +++ & - & - & - \\
\hline B7 & TSC2 & ++ & - & - & - \\
\hline B8 & - & ++ & +++ & - & + \\
\hline B9 & TSC2 & + & + & - & - \\
\hline B10 & TSC1 and 2 & +++ & - & +++ & - \\
\hline B11 & TSC2 & - & +++ & ++ & +++ \\
\hline M8 & - & + & +++ & ++ & ++ \\
\hline
\end{tabular}

and non-neoplastic lesions, because immunohistochemical phenotypes of mTOR-related proteins between a pair of multifocal micronodular pneumocyte hyperplasia lesions were identical in each individual. Second, multifocal micronodular pneumocyte hyperplasia lesions without LOH probably would have uninformative allelic loss or epigenetic events on TSC genes. In fact, a recent study showed inactivation of TCS2 caused by methylation of the TSC2 gene promoter in angiomyolipoma-derived cells. ${ }^{29}$ If so, each of them might be genetically distinct from another one with $\mathrm{LOH}$, but we could not discern whether multifocal micronodular pneumocyte hyperplasia had those events on TSC genes in this study. Finally, we could not completely eliminate the possibility that tissue samples for PCR might be contaminated by normal cells even though our $\mathrm{LOH}$ analysis yielded reliable data. Therefore, we were unable to determine whether multifocal micronodular pneumocyte hyperplasia lesions might be genetically independent from each other, although we showed different LOH patterns between a pair of the lesions from one of eight patients.

Our immunohistochemical data supported the likelihood that all multifocal micronodular

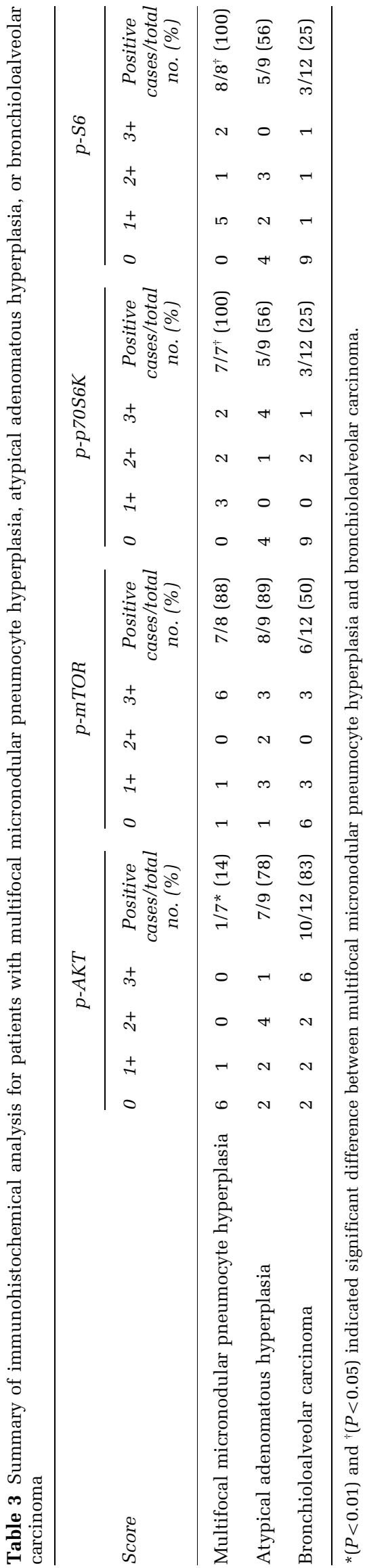


pneumocyte hyperplasia lesions, as well as lymphangioleiomyomatosis and angiomyolipoma, might have a functional loss of TSC genes. ${ }^{25,26}$ Multifocal micronodular pneumocyte hyperplasia cells showed a high frequency of immunopositivity for mTOR pathway-related proteins including p-mTOR, p-p70S6K, and p-S6; that is, downstream proteins regulated by TSC genes. In contrast, all but one patient (M7) lacked immunoreactivity for p-Akt, a regulator of TSC genes. These results indicate that the multifocal micronodular pneumocyte hyperplasia condition might frequently promote constitutive activation of the MTOR signaling pathway caused by a functional loss of TSC genes. Although one patient (M6) included here showed no immunoreactivity for p-mTOR, the multifocal micronodular pneumocyte hyperplasia cells were immunopositive for p-S6 and, consequently, might have undergone constitutive activation of mTOR. In addition, the lack of p-mTOR expression might come from poor fixation of the tissue obtained from autopsy. However, because one patient with multifocal micronodular pneumocyte hyperplasia had immunoreactivity for p-Akt, we cannot exclude the possibility that the mTOR signaling pathway is activated by p-Akt coincidentally with lost function of the TSC2 gene.

We assumed that only the alteration of TSC genes has a role in tumorigenesis but does not directly promote carcinogenesis in pneumocytes. Many genetic alterations in lung adenocarcinomas have been reported including TP53, CDKN2A, STK11, and TSCs as tumor suppressor genes, and KRAS, $E G F R$, and NRAS as proto-oncogenes. ${ }^{30}$ Especially, $K R A S, E G F R$, and TSC genes are associated with the mTOR signaling pathway. These reports suggest that dysregulation of the mTOR signaling pathway by these genes may be involved in carcinogenesis in lung adenocarcinomas. However, few such factors were found in lung adenocarcinomas of patients with TSC who would have TSC germ-line mutations. In fact, in a TSC patient who had TSC1 LOH in both of two multifocal micronodular pneumocyte hyperplasia lesions in this study, a bronchioloalveolar carcinoma lesion did not have LOH on TSC1. In atypical adenomatous hyperplasia or bronchioloalveolar carcinoma, constitutive activation of p-Akt was more frequently detected than TSC LOH, suggesting the limited role of TSC genes in the carcinogenesis of pneumocytes. In addition, in bronchioloalveolar carcinoma patients, mTORrelated proteins should be activated by p-Akt yet were detected less often than in atypical adenomatous hyperplasia patients or, significantly, in multifocal micronodular pneumocyte hyperplasia patients. We have no explanation for these findings, with the possible exception of invoking different pathways of Akt-mTOR signaling. ${ }^{31,32}$ Thus, the preinvasive lesions of pneumocytes might not necessarily occur by gene mutations of TSCs but by dysregulation of other genes including upstream regulatory proteins of the mTOR signaling pathway.
Conversely, patients with TSC are frequently affected with renal cell carcinomas, indicating that functional loss of TSC genes may lead to carcinogenesis in non-pneumocytic cells. ${ }^{33}$ Understanding how alterations of TSC genes produce carcinogenesis in different types of somatic cells will ultimately resolve this issue.

In conclusion, we found in this study that all of eight patients with multifocal micronodular pneumocyte hyperplasia lost TSC gene heterozygosity and for the first time showed loss of both TSC2 alleles. This dysfunction of TSC genes was, accordingly, attributed to constitutive activation of mTOR-related proteins.

\section{Acknowledgements}

We are grateful for Dr Yoichiroh Kobashi (Department of Pathology, Tenri Institute of Medical Research), Dr Chiho Ohbayashi (Department of Pathology, Hyogo Prefectural Cancer Center), Dr Ichiro Yamadori (Division of Clinical Pathology, Okayama Medical Center), Dr Nobukazu Tomiji (Department of Pathology, Iwate Prefectural Central Hospital), Dr Takeshi Fujii (Department of Pathology, Toranomon Hospital), and Dr Hajime Odajima (Division of Pathology, Nishinouchi Branch, Ohta General Hospital) for providing materials of multifocal micronodular pneumocyte hyperplasia, and also many clinicians for providing clinical information of patients with multifocal micronodular pneumocyte hyperplasia. This study was supported by a grant-in-aid for Scientific Research No. 18390243 (Dr Seyama) and No. 19590406 (Dr Kumasaka); in part by the High Technology Research Center grant from the Ministry of Education, Culture, Sports, Science, and Technology, Japan; a grant to the Respiratory Research Group from the Ministry of Health, Labour and Welfare, Japan; and in part by the Institute for Environmental and Gender-Specific Medicine, Juntendo University, Graduate School of Medicine.

\section{Disclosure/conflict of interest}

The authors declare no conflicts of interest.

\section{References}

1 Knudson AG. Chasing the cancer demon. Annu Rev Genet 2000;34:1-19.

2 van Slegtenhorst $\mathrm{M}$, de Hoogt $\mathrm{R}$, Hermans $\mathrm{C}$, et al. Identification of the tuberous sclerosis gene TSC1 on chromosome 9q34. Science 1997;277:805-808.

3 European Chromosome 16 Tuberous Sclerosis Consortium. Identification and characterization of the tuberous sclerosis gene on chromosome 16. Cell 1993;75:1305-1315.

4 Harris TE, Lawrence Jr JC. TOR signaling. Sci STKE 2003;2003:re15. 
5 Jacinto E, Hall MN. Tor signalling in bugs, brain and brawn. Nat Rev Mol Cell Biol 2003;4:117-126.

6 Karbowniczek M, Henske EP. The role of tuberin in cellular differentiation: are B-Raf and MAPK involved? Ann NY Acad Sci 2005;1059:168-173.

7 Popper HH, Juettner-Smolle FM, Pongratz MG. Micronodular hyperplasia of type II pneumocytes-a new lung lesion associated with tuberous sclerosis. Histopathology 1991;18:347-354.

8 Guinee D, Singh R, Azumi N, et al. Multifocal micronodular pneumocyte hyperplasia: a distinctive pulmonary manifestation of tuberous sclerosis. Mod Pathol 1995;8:902-906.

9 Flieder DB, Travis WD. Clear cell 'sugar' tumor of the lung: association with lymphangioleiomyomatosis and multifocal micronodular pneumocyte hyperplasia in a patient with tuberous sclerosis. Am J Surg Pathol 1997;21:1242-1247.

10 Lantuejoul S, Ferretti G, Negoescu A, et al. Multifocal alveolar hyperplasia associated with lymphangioleiomyomatosis in tuberous sclerosis. Histopathology 1997;30:570-575.

11 Muir TE, Leslie KO, Popper H, et al. Micronodular pneumocyte hyperplasia. Am J Surg Pathol 1998; 22:465-472.

12 Yamanaka A, Kitaichi M, Fujimoto T, et al. Multifocal micronodular pneumocyte hyperplasia in a postmenopausal woman with tuberous sclerosis. Virchows Arch 2000;436:389-392.

13 Matsumoto S, Nishioka T, Akiyama T. Renal angiomyolipoma associated with micronodular pneumocyte hyperplasia of the lung with tuberous sclerosis. Int J Urol 2001;8:242-244.

14 Maruyama H, Seyama K, Sobajima J, et al. Multifocal micronodular pneumocyte hyperplasia and lymphangioleiomyomatosis in tuberous sclerosis with a TSC2 gene. Mod Pathol 2001;14:609-614.

15 Franz DN, Brody A, Meyer C, et al. Mutational and radiographic analysis of pulmonary disease consistent with lymphangioleiomyomatosis and micronodular pneumocyte hyperplasia in women with tuberous sclerosis. Am J Respir Crit Care Med 2001;164:661-668.

16 Fujitaka K, Isobe T, Oguri T, et al. A case of micronodular pneumocyte hyperplasia diagnosed through lung biopsy using thoracoscopy. Respiration 2002;69:277-279.

17 Kobashi Y, Yoshida K, Miyashita N, et al. Multifocal micronodular pneumocyte hyperplasia in a man with tuberous sclerosis. Intern Med 2005;44:462-466.

18 Kobayashi T, Satoh K, Ohkawa M. Multifocal micronodular pneumocyte hyperplasia associated with tuberous sclerosis. Acta Radiol 2005;46:37-40.

19 Kamiya $\mathrm{H}$, Shinoda K, Kobayashi N, et al. Tuberous sclerosis complex complicated by pulmonary multinodular shadows. Intern Med 2006;45:275-278.

20 Saito M, Yuasa T, Satoh S, et al. Developing multiple lung nodules in a renal transplant recipient with tuberous sclerosis who had undergone bilateral nephrectomy due to renal cell carcinomas. Int J Urol 2008;15:257-258.

21 Kobashi Y, Sugiu T, Mouri K, et al. Multifocal micronodular pneumocyte hyperplasia associated with tuberous sclerosis: differentiation from multiple atypical adenomatous hyperplasia. Jpn J Clin Oncol 2008;38:451-454.

22 Cancellieri A, Poletti V, Corrin B. Respiratory failure due to micronodular type II pneumocyte hyperplasia. Histopathology 2002;41:263-265.

23 Takamochi K, Ogura T, Suzuki K, et al. Loss of heterozygosity on chromosomes $9 q$ and $16 p$ in atypical adenomatous hyperplasia concomitant with adenocarcinoma of the lung. Am J Pathol 2001;159:1941-1948.

24 Minami Y, Matsuno Y, Iijima T, et al. Prognostication of small-sized primary pulmonary adenocarcinomas by histopathological and karyometric analysis. Lung Cancer 2005;48:339-348.

$25 \mathrm{Yu}$ J, Astrinidis A, Henske EP. Chromosome 16 loss of heterozygosity in tuberous sclerosis and sporadic lymphangiomyomatosis. Am J Respir Crit Care Med 2001;164:1537-1540.

26 Sato T, Seyama K, Fujii H, et al. Mutation analysis of the TSC1 and TSC2 genes in Japanese patients with pulmonary lymphangioleiomyomatosis. J Hum Genet 2002;47:20-28.

27 Smolarek TA, Wessner LL, McCormack FX, et al. Evidence that lymphangiomyomatosis is caused by TSC2 mutations: chromosome 16p13 loss of heterozygosity in angiomyolipomas and lymph nodes from women with lymphangiomyomatosis. Am J Hum Genet 1998;62:810-815.

28 Kumasaka T, Seyama K, Mitani K, et al. Lymphangiogenesis-mediated shedding of LAM cell clusters as a mechanism for dissemination in lymphangioleiomyomatosis. Am J Surg Pathol 2005;29:1356-1366.

29 Lesma E, Sirchia SM, Ancona S, et al. The methylation of the TSC2 promoter underlies the abnormal growth of TSC2 angiomyolipoma-derived smooth muscle cells. Am J Pathol 2009;174:2150-2159.

30 Ding L, Getz G, Wheeler DA, et al. Somatic mutations affect key pathways in lung adenocarcinoma. Nature 2008;455:1069-1075.

31 Dobashi Y, Suzuki S, Matsubara H, et al. Critical and diverse involvement of $\mathrm{Akt} / \mathrm{mammalian}$ target of rapamycin signaling in human lung carcinomas. Cancer 2009;115:107-118.

32 Chiang GG, Abraham RT. Phosphorylation of mammalian target of rapamycin (mTOR) at Ser-2448 is mediated by p70S6 kinase. J Biol Chem 2005; 280:25485-25490.

33 Bjornsson J, Short MP, Kwiatkowski DJ, et al. Tuberous sclerosis-associated renal cell carcinoma. Clinical, pathological, and genetic features. Am J Pathol 1996; 149:1201-1208. 